\title{
VIABILIDAD DE DERMATOFITOS EN MUESTRAS CLINICAS ALMACENADAS
}

\section{(Viability of dermatophytes in stored clinical samples)}

\author{
Zaror, L. ${ }^{1}$ \& Bustamante, $X{ }^{2}$ \\ 'Instituto de Microbiologia Clinica y' 'Escuela de Tecnología \\ Médica: Facultad de Mcdicina. \\ Universidad Austral de Chilc. Casilla 567. Valdivia, Clïle. \\ E-mail: lzarorginaldivia.uca.uach.cl
}

Palabrans clave: Mucstras clínicas. dermalofios. viabilidad Key word: Clinical samples, dermatoplyyles, viability:

\section{RESUMEN}

Con la finalidad de determinar la viabilidad en el tiempo de las estructuras fungicas de dermatofitos. levaduras en mutestras clinicas almacenadas, se estudiaron 257 muestras 22 a 7 años de aniguedad) colectadas entre Enero de 1990 y Diciembre de $199+206$ de uña. 43 de piel y \& de pelor.

El examen microscópico directo se realizi con $\mathrm{KOH}$ -

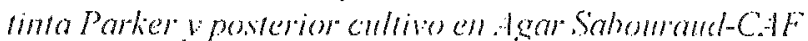

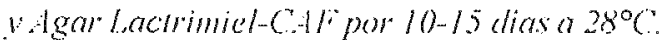

Como controless se analizeron con la misma metordologia, 33 muestras de 5 - $\$$ meses de almacenamiento.

Ein el 100\% de las mueswar control, se observaron elementos fimpicosy solo en el $21,2 \%$ de las almo-

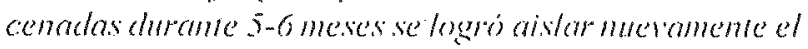
agente causal. La especie más detectada fue T. rubrum.

lat 70, $4 \%$ de las muestras almacenatas per un perior-

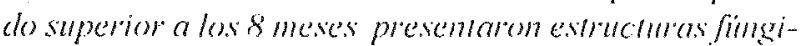
cas al examen microscopico, sin embargo. todens sus culthes resulharom necgativos:

\section{INTRODUCCION}

Los dermatofitos son hongos filamentosos capaces de digerir y obtener nutrientes que reçuicren y ulilizan para su crecimicnto. la queratina de: picl. pelo y uñas (Laronc. 1995). Por ende. las infecciones que producen estin limiladas a los icjidos superficiales (Frecmin. 1989).

Los agcnes etiologicos de las derminofitosis son clasificados en ures géneros animórficos. Microspornm, Trichophyton, Epridermophyton, dependiendo de las calracicristicas de sus macroconidios (Rippon. 1974: Díaren al. 1984: Koneman \& Glon. 1985: Kwon-Cheng \& Bennen. 1092: Laronc. 1995)

\section{SUMMARY}

In order to assess time viability of fungal structures from dermatophyes and veast in clinical stored samples, 257 of them (206 nail, t3 skin and 8 hair samples) collected within. January 1990 and December 1994 were examined (2-7 vears old ) Direct microscopic ohservation was carried out with these samples using KOH-Parker ink and later culture in Sabouraud-CAF agar and Lactrimel-CAF for 10-15 davs at $28^{\circ} \mathrm{C}$.

As a measure of control, 33 samples with a $5-8$ month shorage vere analized as with the same methodology.

The 100\% of control samples exhibited fingal elements whereas in only 21,2\% of samples stored for $5-6$ months it was possible to isolate once more the causing agent. The species mest highly detected was To rubrum.

The 70. to of samples stored for a period greater than 8 monhs reveoted fingal structures under a microscopic exam wet their cullures were negative.

Coincidiendo con la información disponible en Chile las especies frecuentemente implicadas. siguen siendo Trichophy'on mbrum, To mentagrophytes y Microsportum canis, (Zaroret al. 1982. 1988: Dian elal. 1984).

Además. se informan casos de micosis de tipo oportunistas superficiales por Scopulariopsis brevicaulis, Aspergillus candidus y Fusuriun dinnerum (Zaror \&Frick. 1980): Zaror et al. 1984:Negroni. 1984).

En cl diagnóstico de las dermatofitosis debe hacerse sicmpre cramen direclo y cultivo. csic último. permile comprobar cual cs su agenic pallógeno y con ello poder deducir la craluación que pucde tener el proceso. conocer su fuente de origen y establecer cl tratamicnto (Perciro, 1982). 
La sobrevida de dermatolitos cn unias, pelos y picl. ha sido objeto de diversos estudios. Existen algunos trabajos que reconocen la viabilidad de algunos dermatofitos colectados y preservados bajo distimas condiciones (Glass 1948: Kcep. 1960; Rosenthal \& Vunbrouscghem, 1962; Drónk \& Hubalck 1968).

Glass (1948). cncontró que cn pelos infectados con $M$. audouinii, el longo creciat, despućs de estar cmucho on papel durante 479 dias y M. cantis no cha aislido si permanecia almacenado por 348 dias o más.

Keep (1960), logró recultivar tres cepas de $M$. comis aisladas de gatos, después de ser mantenidas en placas ch el laboratorio a temperatura ambientc. La viabilidad de $M$. canis en estos casos de tinca cu felinos fue de $323,315 y$ 422 dias, respectivamente.

Rosenthal \& Vanbreuscghcm (1962). demostmron que algunos dematofilos al ser mantenidos por algún ticmpo, almaccnados cn lubos estériles y envucltos individualmente en papel. pudicron ser reaislados con su morfologia original. Esic cstudio se realizó con sicte cspecics. $T$. violaceum, T.ferrigineum, Tuernicosiun y Tharyanget tuvicron cl más allo porcentaje de sobrevida; T.sudanense y Tyoundei sobrcvivieron bien por dos años. mientas

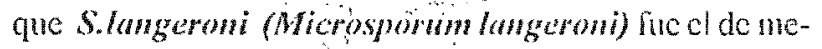
nor riabilidad.

Drórak \& Hubilck (1968). aislaron de cscamas de lesioncs humanas Thentagrophytes, Thmuntagrophyresvar. interdigitale, Trubram y Terermosum, los que fucron prescriados en tubos lapados y mantenidos a tcmpcratura ambicne por 6 a 66 meses. Elincnos viable fie Trubrum, ya que no pudo ser reaislado de nimguna de has muestas con más de 6 mescs de almacenamicnto. Al contrario $T$.

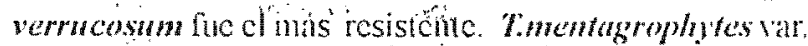
inter digitale mostró una viabiliálid menor a 9 meses, micntras que Tomentagrophyles se reaisló después de 15 meses. Ningin dermanofito fuc capaz de orecerdespués de un petiodo de almacenaje superior a 20 meses (Drórak y Hubalck. 1968 ).

La información acerca de viabilidad de dermmofitos en mucstras clinicas es limitada c insuficicnte sin cmbargo. los dalos disponibles en ia literatura coinciden en que la mayoria de estos licnen una sobrevida limitada cn miucstrasclinicas.

Nuestro objetivo fue csludiar la viabilidad de dermatofitos presentes on mucstras clinicas ahmacenadas por periodos prolongados:

\section{MATERIAL Y METODOS}

Se estudiaron 257 muestras clinicas obenidas de pacientes atcudidos en el Instituto de Microbiología Clinica de la Universidad Austral de Chile. cntre Encro 1990 y Diciombre de 1994.
Las mucstras fucron procesadas originalmente en ese periodo, mediunte examen microscópico dirccio y cultiro: posteriomente se almacenó el remanente de la muestra clínica (raspado de picl, uñts y pelos) cntre dos portaobjcios envuclos en papel.

Del total de mucsiras, 206 correspondicron a tinas, 43 a picl y 8 a pelos y considerando el liempo de inicio de nuestra imestigación. se establece que las muestras a examinar luieron un tiempo múnino de almacenamiento de 2 años y un máxino de 7.

Como controles positivos para la viabilidad, se sembraron 33 muestras cuyo ticmpo de almacenaje fue de solo 5-8 meses. Estas fueron recolectadas entre Encro y Noviembre de 19\%6, esta última marca el inicio de nucstra investigación.

Para el examen microscópico direclo de cscamas de picl, uñts y pelos. ćstas se trataron con $\mathrm{KOH}$ al $10 \%$ más tinta Parker 51 azul permanchte y $\mathrm{KOH}$ al $10 \%$ aldicionado de Chloroblack E (Burke \& Joncs. 1984).

El csudio tambićn incluyó la obsenación y cultwo de lovaduras en las muestras clinicas almacenadas. Para la observación microscópica de las células levaduriformes fue necesario dejar las prepanaciones con KOH al 10\% Chloroblack en cámara humeda por $2+$ horas. para una meior observación.

Todas las mucsuas rucron sembradas en Agar Sabouraud dextrosido, Agar Lacirimiel (adicionados de cloranfenicol levógiro) c incubadas a $28^{\circ} \mathrm{C}$ por 15 días. antes de considerarlas neganivas.

\section{RESULTADOS}

En la Tabla 1, se prescnam los hallazgos on 33 mucstras clinicas con un período de almaconmiento no inferior a 5 ni superior a 8 meses. El mavor numero lo representan las micstras de una $(51.5 \%)$ y piel $(42,4 \%)$.

Tablat.

Hongos previamente aislados en muestras control según localización

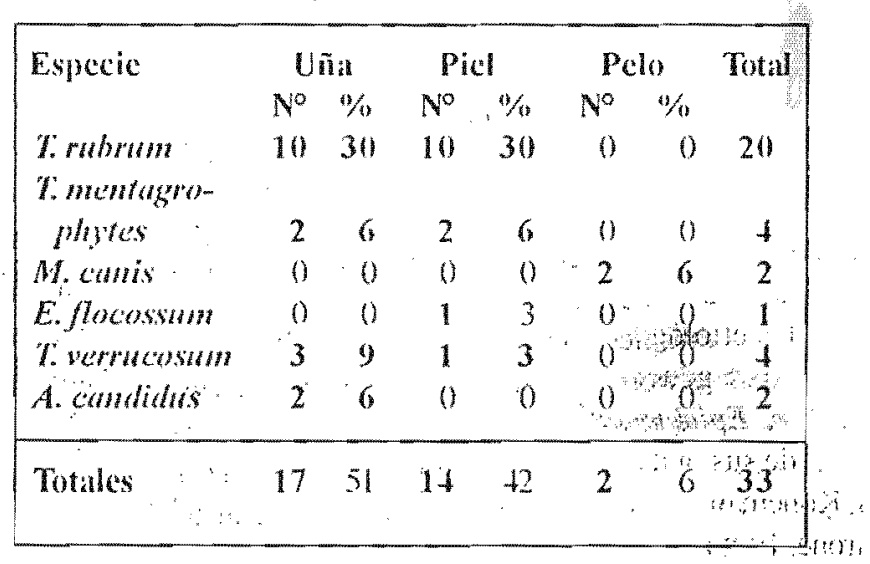


En la Tabla 2. seaprecian los hallazgos al examen microscópico dirceto de hongos filmentosos y levaduriformes a partir de muestras a maconadas entre 1900-1994. El miyor nimero de positividad se obturo mevamente cn thã con un $53,7 \%$ del tolal de las mucst rus $+5 \%$ para

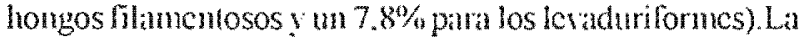
lotalidad de los cullivos fre negatina en anbos medios.

En la Tabla 3. se presenta cl lipo de muestraversus los hallages a microscopia direch y cultivo de his mucstras almacenadas che 5-8 meses. Ala microscopia, sc obsenaron clementos fúngicos en el $100 \%$ de las mucstras y cu ol $21,2 \%$ de cllas se aislaron: Trubrom $(15,2 \%)$. M.cumis (3\%) y Aspergilhs candidus (3\%)(Tablat).

El mayor mimero de posilividad en los cultivo sc obtuvo cn his muestras almaccnadas durame 5 meses. Por sobre esle tiempo se negalivizaron. con cxcepción de una donde se aisló Trubram en una muestra de 6 meses de anigucdad.

Tabla 2.

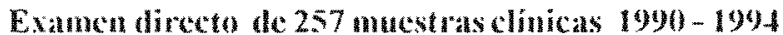

\begin{tabular}{|c|c|c|c|c|c|c|c|c|}
\hline \multicolumn{5}{|c|}{ Honges filamentosos } & \multicolumn{4}{|c|}{ Homges levaduriformes } \\
\hline \multirow[t]{2}{*}{ Muestrat } & \multicolumn{2}{|c|}{ Poniliva } & \multicolumn{2}{|c|}{ Nengativo } & \multicolumn{2}{|c|}{ Positivo } & \multicolumn{2}{|c|}{ Netentivo } \\
\hline & No & $11 / 4$ & No & $\%$ & $N^{\circ}$ & $\%$ & $N^{\circ}$ & $0 \%$ \\
\hline Unia & 118 & 45 & 12 & 4.7 & 20) & 7.8 & 56 & 21.8 \\
\hline Picl & 35 & 136 & 3 & 1.2 & 2 & 0.8 & 3 & 1.2 \\
\hline Pelo & 8 & 3.1 & 0 & 0 & 0 & 0 & 0 & 0 \\
\hline Total & 161 & 62,6 & 15 & 5,9 & 22 & 8,6 & 59 & 23 \\
\hline
\end{tabular}

Talblak 3.

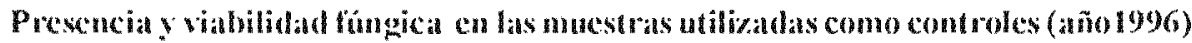

\begin{tabular}{|c|c|c|c|c|c|c|c|c|c|}
\hline \multirow{3}{*}{ Mucstral } & \multicolumn{4}{|c|}{ Examen directo } & \multicolumn{4}{|c|}{ Cullivo } & \multirow{3}{*}{$\begin{array}{r}\text { Totall } \\
\%\end{array}$} \\
\hline & \multicolumn{2}{|c|}{ Posilivo } & \multicolumn{2}{|c|}{ Negativo } & \multicolumn{2}{|c|}{ Positiva } & \multicolumn{2}{|c|}{ Negativan } & \\
\hline & $N^{\circ}$ & $3 / \%$ & $\mathrm{~N}^{\circ}$ & $8 / 4$ & $N^{0}$ & $\%$ & No & $\%$ & \\
\hline Una & 17 & 515 & 0 & 0 & + & 12.1 & 13 & 394 & 17 \\
\hline Picl & $H$ & 12,4 & () & 0 & 2 & 6.1 & 12 & $36+$ & $H$ \\
\hline Pclo & 2 & 61 & 0 & 0 & 1 & 30 & 1 & 30 & 2 \\
\hline Total & 33 & 100 & 0 & 0 & 7 & 21,2 & 26 & 78,8 & 33 \\
\hline
\end{tabular}

Tablat.

Hongus reashalos be muest ras cont rol segun origen y ticmpo de almacenaje

\begin{tabular}{|c|c|c|c|c|c|c|c|c|c|}
\hline \multirow{2}{*}{$\begin{array}{l}\text { Aislamiculo } \\
\left(N^{\circ}\right)\end{array}$} & \multirow[t]{2}{*}{ Origen } & \multirow[t]{2}{*}{$\begin{array}{l}\text { Thompo } \\
\text { Almatowalic }\end{array}$} & \multicolumn{2}{|c|}{$\begin{array}{c}\text { No cultivas } \\
\text { Pasilivo }\end{array}$} & \multicolumn{2}{|c|}{$\begin{array}{l}\text { No cultivas } \\
\text { Neativas }\end{array}$} & \multirow[t]{2}{*}{ Total } & & \multirow{2}{*}{$\begin{array}{l}\% / 6 \\
\text { Espuccic } \\
\text { aivlada }\end{array}$} \\
\hline & & & & $\%$ & & $B / 4$ & & $\%$ & \\
\hline T. rubrum (10) & Unin & $5: 6$ & 3 & 9.1 & 7 & 21.2 & 10 & 30,3 & 9.1 \\
\hline (11) & Picl & 5 & 2 & 6.1 & 8 & $2+.2$ & 10 & 30.3 & 6.1 \\
\hline A. condidus (2) & Uni: & 5 & $i$ & 30 & 1 & 30 & 2 & 60 & 30 \\
\hline M.conis (2) & Pclo & 5 & 1 & 3.11 & 1 & 30 & 2 & 60 & 30 \\
\hline Total & & & 1 & 21.2 & 17 & 51,4 & 24 & 72,6 & \\
\hline
\end{tabular}




\section{DISCUSION}

En los diagnósticos micológicos de las 257 muestras clínicas procesadas entre los periodos $1990-1994$, los principales agentes de dermatomicosis, especialmente de adultos, fueron Trubrum $(39,7 \%)$ y T. mentugrophlytes $(16,5 \%)$. De los hongos levaduriformes, C. albicans $(23,9 \%)$ fiue el agente más inportante en las onicomicosis con compromiso periungueal de la mano, lo que coincide con los esiudios realizados por Zaror el al. (1982). López \& Rivera (1984) y Canteros (1993).

El examen direclo es esencial cu el diagnóslico de una micosis, debido a que por modio de éste se establece en forma rápida y efecliva la presencia fúngica en la muestra, orientando al clinico acerca de la lerapia a seguir (Ausina, 1982). Sin embargo, como csic cxamen no permite discriminar hongos viables o no, el cultivo se hace indispensible.

En las publicaciones disponibles se registran estudios de viabilidad realizados principalmente en muestras de piel y pelos, que van de dias a varios meses (Class, 1948; Keep, 1960; Roscnthal \& Vanbrouseghen, 1962, Dvorak \& Hubalck, 1968). En mucstro csludio no sc logró aislar ningún dermatofito debido al tiempo de almaconạc que fluctuó entre los 2 y 7 años. guizís demasiado ticmpo para la sobrevivencia de las diversis estructuras fúngicas bajo condiciones ambicntales adversas(pérdida de agua cspecialmente). Es posible que algunas de cllas pudicran mantenerse aún viables en ticmpos inferiores a los 2 anos. situación que no consideramos y que mercecrial posteriores estudios.

Keep (1960), publicó la viabilidad de ires cepas de $M$. canis, colectadas de tres casos separados de tiñas de ga105; el tiempo de almacenaje registrado fue de $315,323 \mathrm{y}$ 422 dias.
Aunque los tiempos de almacenaje y las especies aisladas varian en los datos aportados por difcrentes autores, todos concuerdan en la forma y las condiciones de almaceninje. En la litenutura se registran reaislamientos en 20 meses de Toverrucosum en escamas de piel (Dvórak \& Hubalck, 1968); después de 5 años de Trviolaceum, $T$. ferruginetum, Tverrucosum y Thuryangei ( $=$ Thnegninii) en pelos (Rosenthal y Vanbreuseghicm, 1962) y de $A$. andoninia y M.lanosunt (=M.canis) en pelo después de $121+y 145+$ dias respectivamente (Glass, 1948).

En las muestras controles almacenadas entre $5-8 \mathrm{me}$ ses, sólo lubo cullivos posilivos de dermatofitos en un $21,2 \%$ y en gencral la sobrevivencia fúngica no sobrepasó los 5 meses. Estos resultados coinciden con los registrados por Diórak y Hubalck (1968) para muestras de piel.

Keep (1960). hace mención de la importancia que tendrian los pelos de galo infectados, como reservorio de material infectado, pudiendo asi contaminar el medio ambicnte por al menos 13 meses, ticmpo míximo de almacenaje de las nuestras, en que logró reaislar cl agente productor de la micosis.

M.cumis, cspecic zooantropofilica, debería ser capaz de soportar mayores tiempos de almacenaje ( superiores a 6 meses), por la producción de sus diversos propágulos infectantcs, siluación que no pudo comprobarse, quizás debido al reducido múmero de mucstras control que lo conicnian (2) asi como en las muestras analizadas (9) y al amplio periodo de almacenaic.

La mayoria de las especies imolucradas en nuestro estudio control. fucron capaces de soportar periodos de latencia de hasta 6 meses sin perder su viabilidad demostrando así los alcances cpidcmiológicos de algunos dermatofitos u hongos oportunistas en muestras clinicas.

\section{REFERENCIAS}

1.- Ausina, R. V(1982). Diagnosico micologico de las dermatufitosis. En : IM.Torres.R.(Ed.). Dermatolitos y dermattofitosis. Laboratorio Dr. Exteve S.A. Barcelona. pp. 85-25

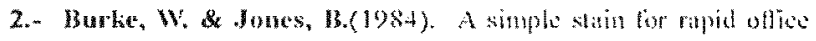
diagnesisis of thingus infection of the shin. Arthises of Dematolygy $120: 1519-1520$

3.- Canteros, C.E.; Davel,C.O.; Vivot, W; W'Amico, S. (1993). Incidencia de los distinos agems stiohogicos de micosis superticisles. Rev. Arg. Micologia 25:1294135

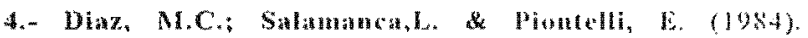
Dermatofitosis: Cn problema del pasads. wn desilio del presente. Adelantos en Nicrobiol. $y$ Ent. Infese 3:212-273

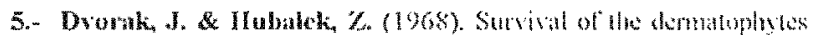

in human skin seales. Ardites Dormalology: $28: 540-542$

6." Freman, 1B.A. (1989). Micologia Midicat Hongos y

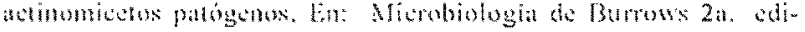

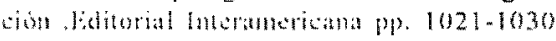

7.- Clas, f. (1948). A viabilit of lungus in hairs from pations with thea eapitis. Areh of Dematol. 57:122-124

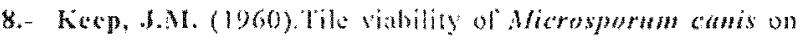
isolated wh hair the Australiantet. Isume $36: 277-278$

9.- Kinneman, L.W, \& (iten, D.R. (1985). Identilication de

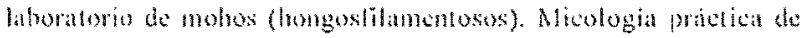

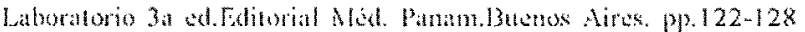

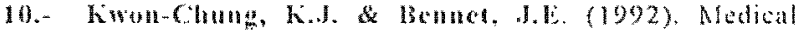


Mroology: Lditom Lan and Pebiger. Philadelhit.

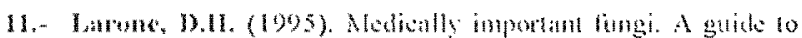

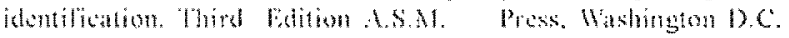

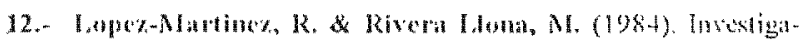

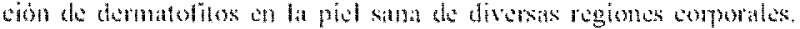
Rot latt. Anmer. Microbiol. $25365-369$

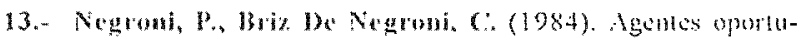

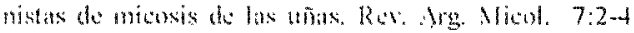

14.- Pereiro, M.(1982). Importaticia de los dermatolitos en pato-

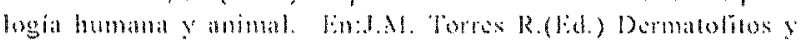

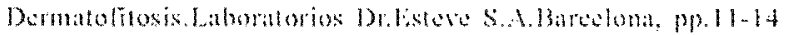

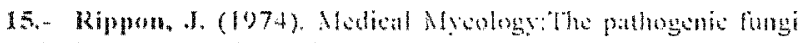

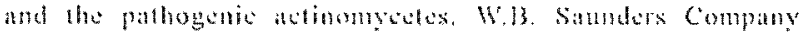
Ptrilutsilphit.

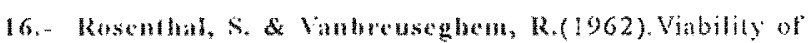
the dermophyte in depilated hairs Arch. Dermatol. 85:103-105

17.- Zaro, I.; Casas, S; Mamu, R, Thibol, J; Fischman, 0 . (19ss). Dematofios en perres y gatos sanos en Valdivia. Chile. Arch. Nod Vet $20: 140,143$

18.- Zarer, I. \& Vuth 1". (1980). Onicomicosis por Scophlariopsis brewismalis. Rev, Med. Chile 108:721-723

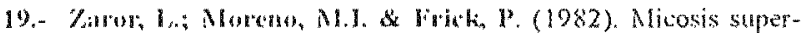

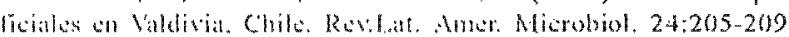

20.- Zaron, L.; Morem, MAl; Frick, Pa; Negrom, N. (1984).

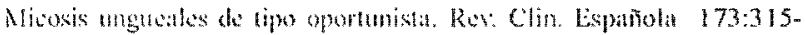
316 